\title{
First results of systematic studies done with different types of Silicon Photomultipliers
}

\author{
C. Bosio ${ }^{1}$, S. Gentile ${ }^{2}$, E. Kuznetsova ${ }^{1}$, F. Meddi ${ }^{2}$ \\ 1- INFN Roma 1 \\ Piazzale Aldo Moro 5, 00185 Roma, Italy \\ 2- Università degli Studi di Roma "La Sapienza" \\ Piazzale Aldo Moro 5, 00185 Roma, Italy
}

\begin{abstract}
The presented results are obtained during the first steps taken in order to develop a setup and measurement procedures which allow to compare properties of diverse kinds of silicon photomultipliers. The response to low-intensity light was studied for silicon photomultipliers produced by CPTA (Russia), Hamamatsu (Japan), ITC-irst (Italy) and SensL (Ireland).
\end{abstract}

\section{Introduction}

Fast development of the silicon photomultiplier technology results in a number of different sensor types available on market and produced by various manufacturers. Despite the different commercial names of the devices, here all of them are referred as SiPM.

The measurements discussed here include current-voltage characteristics and studies of the SiPM response to low-intensity light. The results of the former measurements are mostly used to check the sample operability and to define the range of bias voltages for the latter studies. The measurements of the SiPM response to the light provide a number of parameters suitable for the comparison of different samples.

Five samples of silicon photomultipliers produced by the following manufacturers have been studied: CPTA HAMAMATSU produced Multi-Pixel Photon Counter S10362-11-025C 1, ITC-irstG and SensL produced samples were compared on the base of the measurement results.

\section{Measurement Setup}

In order to obtain the SiPM response to low-intensity light, the sensors were illuminated with a light emitting diode operated in a pulse mode. The LED drive developed by Institute of Physics ASCR (Prague) was used. The drive provides current pulses with tunable amplitude and duration with a sharp rise time down to $2 \mathrm{~ns}$ [2]. The signal from SiPM was read out with a charge-sensitive preamplifier and digitised with an integrating ADC. The LED pulse of about 6 ns duration and ADC gate of 65 ns width were synchronised by means of a common trigger.

The measurements were done at room temperature. Temperature variation during the measurements done for one sample did not exceed $2^{\circ} \mathrm{C}$, for all measurements discussed here the total variation was less than $4^{\circ} \mathrm{C}$.

${ }^{a}$ CPTA, Russia, http://www.zao-cpta.ru

${ }^{b}$ Forimtech SA, http://www.forimtech.ch

${ }^{\mathrm{c}}$ ITC-irst, Italy, http://www.itc.it/irst

${ }^{\mathrm{d}}$ SensL, Ireland, http://www.sensl.com

LCWS/ILC 2007 


\section{Measurement Results}

Fig. 1 shows an example of the SiPM response to low-intensity light measured with the described setup. The peaked structure indicates the number of cells fired during one light pulse, starting with the pedestal for no cells fired. The distance between peaks corresponds to the SiPM gain.

The spectrum is fitted as a sum of gaussian distributions:

$$
\sum_{i} G\left(N_{i}, \mu_{i}, \sigma_{i}\right)=\sum_{i} G\left(N_{i}, \mu_{0}+i \cdot g, \sigma_{i}\right),
$$

where $\mu_{0}$ corresponds to the pedestal position and $g$ is gain in units of $\mathrm{ADC}$ counts. From statistical considerations the width of $i^{\text {th }}$ peak $\sigma_{i}$ can be expressed as

$$
\sigma_{i}=\sqrt{\sigma_{0}^{2}+i \cdot\left\langle\sigma_{p x}\right\rangle^{2}}
$$

where $\sigma_{0}$ is the pedestal width and $\left\langle\sigma_{p x}\right\rangle$ represents fluctuations of the one-cell response averaged over the active area of the sample.

The gain as a function of the overvoltage $U_{b i a s}-U_{b r d}$ was studied for several values of the bias voltage. The breakdown voltage $U_{b r d}$ is defined here as the bias voltage corresponding to the gain equal to one. The results are shown in fig. 2 (left). Fig. 2 (right) shows the dark current as function of the overvoltage.
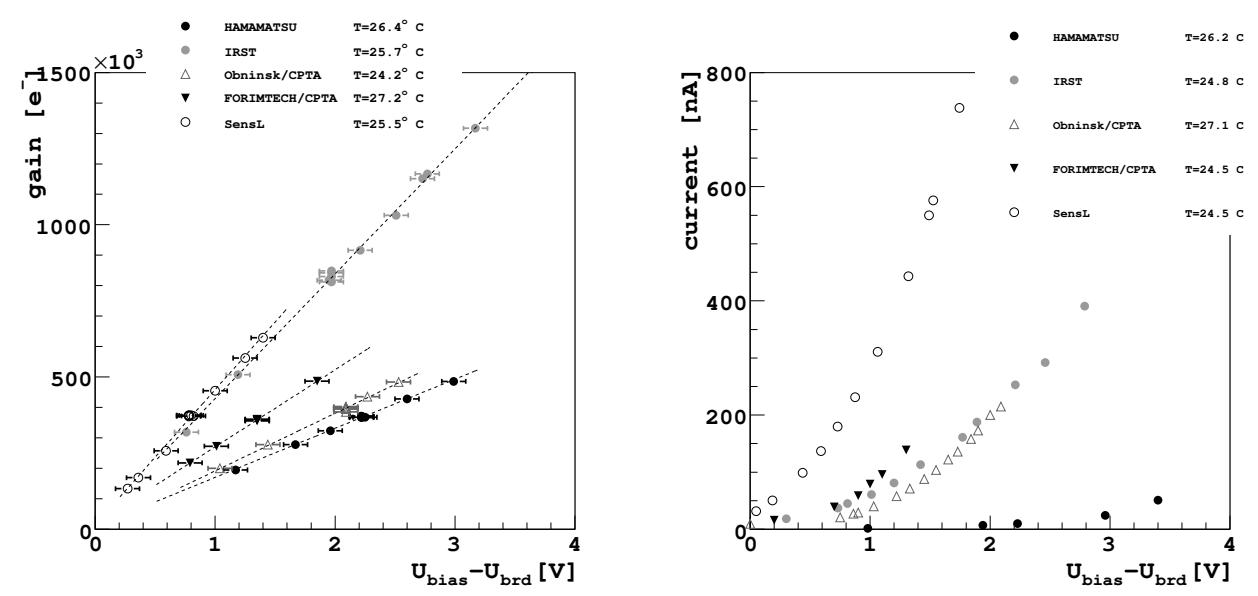

Figure 2: Gain (left) and dark current (right) as a functions of the overvoltage.

Fig. 3 shows gain normalised to the corresponding values of pedestal width $\sigma_{0}$ (left) and $\left\langle\sigma_{p x}\right\rangle$ (right) as functions of the overvoltage. Being averaged over the active area of SiPM, 
$\left\langle\sigma_{p x}\right\rangle$ contains statistical and systematic parts:

$$
\left\langle\sigma_{p x}\right\rangle \sim \sqrt{N+\sigma_{n u}^{2}}
$$

where $N$ is the average number of charge carriers in the avalanche and $\sigma_{n u}$ represents nonuniformity of the amplification over the SiPM active area. Since gain is proportional to the carriers number, $g \sim N$, the ratio $g /\left\langle\sigma_{p x}\right\rangle$ shown in fig. 3 (right) tends to a linear behaviour for the low gain values $N<<\sigma_{n u}^{2}$. For the high gain values $N>>\sigma_{n u}^{2}$ the dependence would correspond to square root low $g /\left\langle\sigma_{p x}\right\rangle \sim \sqrt{N}$ in the case of a constant $\sigma_{n u}$. As seen from fig. 3 (right), the ratio $g /\left\langle\sigma_{p x}\right\rangle$ obtained for some of the samples indicates growth of the non-uniformity factor $\sigma_{n u}$ with the bias voltage.
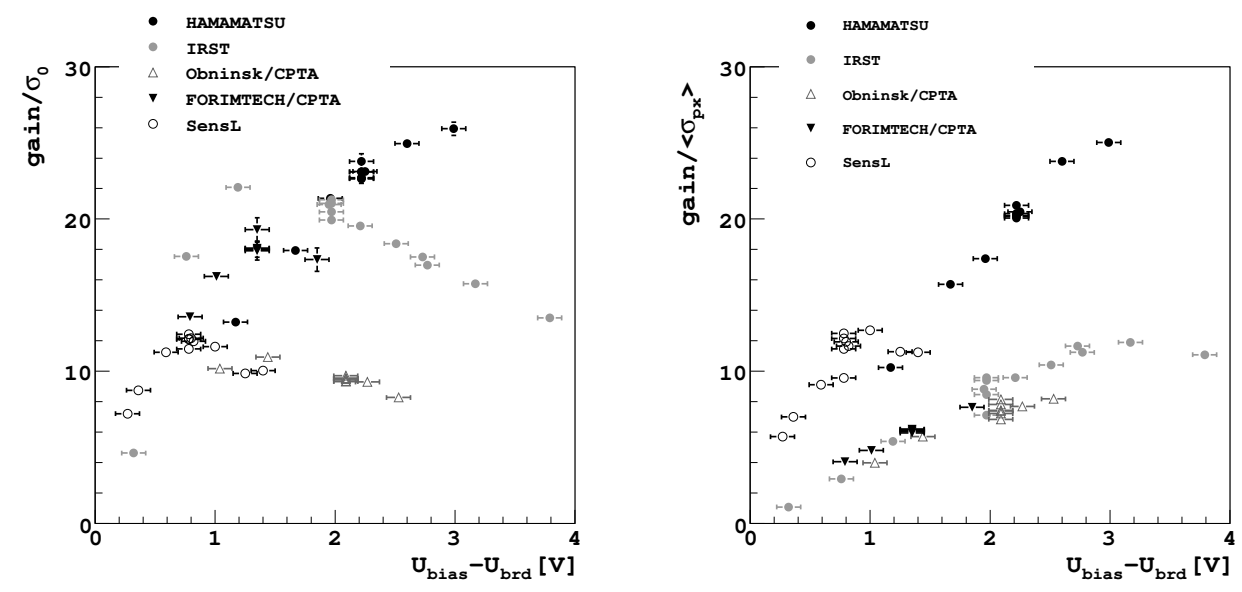

Figure 3: The gain normalised to the corresponding values of pedestal width (left) and $\left\langle\sigma_{p x}\right\rangle$ (right) as functions of the overvoltage.

\section{Conclusion}

The obtained results demonstrated operability and potential of the developed setup. Further development and tune of the setup, measurement procedure and data treatment will allow to obtain comparative characteristics of diverse types of silicon photomultipliers.

\section{Acknowledgements}

We would like to thank Prof. R. Battiston (University and INFN of Perugia), Prof. V. Saveliev (Obninsk State University and DESY), Dr. I. Polak (Institute of Physics of the ASCR, Prague), Dr. E. Popova (MEPhI) and Mr. N. D‘Ascenzo (DESY) for their support and fruitful discussions.

\section{References}

[1] HAMAMATSU PHOTONICS K.K, Product catalogue No. KAPD0002E02, 2007.

[2] I. Polak. Talk at ILC Workshop, Valencia, 2006. 